\title{
LAS MUJERES TRABAJADORAS DE NUEVO LEÓN A PRINCIPIOS DEL SIGLO XXI, ¿AVANZANDO HACIA LA IGUALDAD? UN ANÁLISIS DESDE LA PERSPECTIVA DEL TRABAJO DECENTE
}

\section{Elizabeth Gálvez Santillán}

Instituto de Investigaciones Sociales (IINSO). Universidad Autónoma de Nuevo León, México. elizabethglvez@yahoo.com.mx Gabriela Monforte García

Tecnológico de Monterrey, Campus Monterrey, gmonforte@itesm. $\mathrm{mx}$

\section{Resumen}

El avance hacia la sustentabilidad desde la perspectiva del Trabajo Decente, de la Organización Internacional del Trabajo, implica no solo una suficiente oferta de trabajo para todas y para todos, sino también, la igualdad en salarios, prestaciones y seguridad social. La erradicación de la pobreza sólo será posible cuando un empleo de calidad permita a todos los sectores de la población tener un empleo que asegure un nivel de vida digno. En este artículo se realizó una investigación sobre la calidad del empleo de las mujeres trabajadoras en el estado de Nuevo León a través del el Índice de Trabajo Decente. El Índice se calculó a partir de los siguientes indicadores: el empleo durable, el salario suficiente y las prestaciones sociales o equivalentes. Bajo el criterio establecido por el Programa de las Naciones Unidas para el Desarrollo, para la interpretación del Índice de Desarrollo Humano, los resultados mostraron en resumen que en 2005 las condiciones laborales de las mujeres trabajadoras se encontraban en un nivel medio de trabajo decente, mientras que en 2016 las condiciones laborales de las mujeres en Nuevo León disminuyeron a un nivel bajo de trabajo decente. En conclusión, este análisis muestra que desde la visión del trabajo decente y del desarrollo sustentable un aspecto al que se le debe dar más importancia desde las políticas públicas en el Estado es a los ingresos que reciben las mujeres trabajadoras. 
Palabras clave: equidad, ingresos, bienestar

\section{WOMEN WORKERS OF NUEVO LEÓN AT THE BEGINNING OF THE 21ST CENTURY, ADVANCING TOWARDS EQUALITY? AN ANALYSIS FROM THE PERSPECTIVE OF DECENT WORK}

\section{Abstract}

The progress to sustainability according to the International Labor Organization, and their Decent Work Index, implies not only a sufficient labor offer for everyone, but also, equal salaries, benefits, and social security. The end of poverty, will only be possible when people from every sector of the population has access to quality employment that can assure them a dignifying lifestyle. In this article, an investigation about the quality of employment of working women in the state of Nuevo León is presented, measured through the decent Work Index. Durable employment, sufficient salary, and social benefits are the indicators taken into consideration in order to calculate the Index. Under the criteria established by the United Nations Development Program for the interpretation of the Human Development Index, the results show that in Nuevo Leon from the year 2005 to the year 2016 the labor conditions of working women went from medium Decent Work, to low Decent Work. In conclusion, this analysis shows that through the vision of Decent Work and Sustainable Development, working women's salary is an aspect that should be taken into consideration in the state's public politics.

Keywords: equity, income, wellness

\section{Introducción}

Los objetivos de desarrollo sostenible 2030, señalan que la meta de la sustentabilidad podrá alcanzarse en la medida en que se reduzca la pobreza y se consiga mejorar la calidad de vida de las personas. La erradicación de la pobreza solo será posible mediante empleos estables y bien remunerados; no obstante, el desempleo está aumentando a nivel mundial siendo los más afectados las mujeres y los hombres jóvenes (ONU, 2015). De acuerdo a cifras del 
Instituto Nacional de Estadística y Geografía (INEGI), en el 2015, Nuevo León tiene el séptimo lugar en cuanto a empleo generado en el país, con 2.2 millones de personas ocupadas, de las cuales el $37 \%$ son mujeres. La participación de la fuerza laboral femenina ha ido en aumento al paso del tiempo, en el 2005 la proporción era de un 35\%, lo que demuestra que cada vez más las mujeres están ganando espacio en las actividades económicas de la entidad, incluso en el año de referencia la tasa de desempleo femenina era igual a la masculina de 5\% (INEGI, 2016). Siguiendo esta reflexión de una participación creciente de la mujer en el empleo, se esperaría que la calidad de los mismos fuera cada vez de mayor.

Anker, Chernyshev, Egger, Mehran \& Ritter (2003), señalan que el trabajo decente, es un fin importante por derecho propio, ya que hablar de éste es referirse a una faceta esencial de la vida debido a que es algo a lo que dedicamos gran parte de nuestro tiempo y es un pilar para la integración social y la autoestima de las personas. Contar con la oportunidad de acceder a un trabajo decente constituye un acto de libertad que la sociedad y sus instituciones son responsables de proveer para lograr el desarrollo. Para realizar el análisis sobre la calidad del empleo de las mujeres trabajadoras en Nuevo León se utilizará el enfoque de trabajo decente de la Organización Internacional del Trabajo (OIT), el cual ha sido tomado por la Organización de las Naciones Unidas (ONU), como uno de los objetivos de la Agenda 2030 para el Desarrollo Sustentable. El documento se organiza de la siguiente manera: en la primera parte llamada marco teórico se hace una reflexión sobre la calidad del empleo desde la visión del trabajo decente y el desarrollo sustentable; posteriormente se presenta el método apartado donde se describe el proceso a través del cual se estimarán los indicadores que permitirán evaluar el trabajo decente de las mujeres en el estado de Nuevo León; después se presentan los resultados obtenidos y, por último, las conclusiones.

\section{Marco Teórico}

\section{El trabajo decente}

Tras reconocer que las fuerzas del mercado actúan de manera desigual en la sociedad, incluso en el mercado laboral, la Organización Internacional de Trabajo (OIT), adoptó en su 87ª Reunión, 
en el año de 1999, el Programa de Trabajo Decente, como el objetivo principal de la organización. Este concepto se definió como el "trabajo productivo en condiciones de libertad, equidad, seguridad y dignidad, en el cual los derechos son protegidos y que cuenta con remuneración adecuada y protección social" (OIT, 1999: 4).

Para alcanzar este propósito se establecieron cuatro objetivos estratégicos: a) la promoción de los derechos fundamentales en el trabajo; b) la promoción del empleo; c) la protección social contra las situaciones de vulnerabilidad; y d) el fomento del diálogo social (OIT, 1999).

Es decir, la noción de trabajo decente se integra por los siguientes componentes (OIT, 1999: 15-16): "trabajo productivo; con protección de derechos; con ingresos adecuados; con protección social y; con presencia del tripartimos y del diálogo social”. El concepto de Trabajo Decente trascendió de tal manera que en el Informe sobre el Desarrollo Humano del Programa de las Naciones Unidas para el Desarrollo (PNUD) del 2000, se señaló que una de las libertades del ser humano, es la de tener un trabajo decente, sin explotación, ya que permite a la gente adquirir los bienes y servicios que necesita, potencia socialmente a las personas, aumentando su dignidad y autoestima y puede ayudar a la gente a participar políticamente, permitiendo con ello influir en las decisiones en el lugar de trabajo y fuera de él. Así mismo, en el documento se indicaba que el trabajo decente no sólo es una meta del desarrollo sin también es un derecho humano (PNUD, 2000).

El concepto de trabajo decente se elevó a un nivel de prioridad internacional, regional y nacional, como se puede observar en el documento final de la Cumbre Mundial 2005, de las Naciones Unidas, donde los dirigentes definieron el trabajo decente como un objetivo nacional y mundial para lograr una globalización justa y como parte del esfuerzo para cumplir los Objetivos de Desarrollo del Milenio, pues cada vez más la comunidad internacional que se ocupa del desarrollo se da cuenta que el trabajo decente es el camino para disminuir la pobreza. Ahora, en el 2015, el trabajo decente se convirtió en uno de los objetivos de la Agenda 2030 para el Desarrollo Sostenible. 


\section{El trabajo decente y el desarrollo sustentable}

Uno de los temas que ocupan a las ciencias sociales es comprender cómo se puede dar el desarrollo de las sociedades desde una visión más integral, es decir, que incluya no sólo el aspecto económico sino también otras dimensiones que son importantes para todo ser humano. Es así como entre los años de 1950 y 1960, comienzan a surgir una serie de propuestas sobre el desarrollo, por ejemplo, la teoría estructuralista de la Comisión Económica para América Latina (CEPAL), el pensamiento ambientalista, la teoría de la regulación, entre otras. Sin embargo, es hasta 1987 cuando la Comisión Mundial del Medio Ambiente del Desarrollo (CMMAD), de las Naciones Unidas, entonces dirigida por la Dra. Gro Harlem Brundtland, dio a conocer el informe "Nuestro Futuro Común", haciendo con esto una propuesta conceptual sobre el desarrollo sustentable (Gutiérrez \& González, 2010). En el Informe Brundtland se define desarrollo sustentable como el "Desarrollo sustentable es el desarrollo que satisface las necesidades del presente sin comprometer la capacidad de las generaciones futuras para satisfacer sus propias necesidades" (CMMAD, 1987: 24).

De acuerdo a esta visión, para lograr el desarrollo sustentable se requiere elaborar políticas que permitan cambiar el rumbo del desarrollo, entre ellas: revitalizar el crecimiento económico, cambiar la calidad del crecimiento económico, satisfacer las necesidades humanas esenciales y conservar y mejorar la base de los recursos. Es precisamente en el objetivo de la satisfacción de las necesidades humanas esenciales, donde el informe hace referencia que el principal reto del desarrollo es satisfacer las necesidades de la población, siendo la más básica el que cuenten con un medio de vida, es decir, con un empleo el cual debe representar una oportunidad sostenible de trabajo ya que debe de ser capaz de permitir satisfacer los estándares mínimos de consumo de los hogares. El concepto de trabajo decente coincide con esta preocupación del desarrollo sustentable ya que la población no podrá mejorar su calidad de vida si no cuenta con un empleo de calidad, es decir, con un empleo que le permita salir de la pobreza y tener al menos un nivel de vida mínimamente digno. Es por esto que: "un número mayor de personas con empleos decen- 
tes significa un crecimiento económico más fuerte e inclusivo, y mayor crecimiento significa más recursos disponibles para crear empleos decentes" (OIT, 2016: 2).

De acuerdo a Anand \& Sen (1994), la propuesta del desarrollo sustentable se presenta como un nuevo estilo que evoca el principio de universalismo, al hacer referencia que el desarrollo debe de ser para todos y para todas y para ello se requieren nuevas formas de cooperación solidaria entre los seres humanos. En su definición, señala la cuestión inter generacional porque se preocupa por la satisfacción de las necesidades de las generaciones futuras, así como intra generacional porque destaca la importancia de eliminar la pobreza en las actuales generaciones para que de esa forma puedan satisfacer al menos sus necesidades humanas básicas que les permita disfrutar de una vida mejor, todo esto respetando el medioambiente. La definición de desarrollo sustentable del Informe Brundtland aún se mantiene como la base en la discusión del desarrollo a largo plazo en el mundo, esta se apoya en tres pilares fundamentales: el desarrollo social, el desarrollo económico y el cuidado del medio ambiente y es el fundamento, junto con el Desarrollo Humano, de la Agenda 2030 del Desarrollo Sustentable (ONU, 2016).

\section{La agenda 2030 para el Desarrollo Sostenible y el Trabajo Decente}

La Agenda 2030, para el Desarrollo Sostenible, la cual fue adoptada por los líderes del mundo en una cumbre de las Naciones Unidas realizada en septiembre del 2015 en Nueva York, está constituida por 17 Objetivos con 169 metas que abarcan las dimensiones económica, social y medioambiental de Desarrollo Sostenible (ONU, 2015a). Su meta principal es erradicar la pobreza del mundo:

Estamos resueltos a poner fin a la pobreza y el hambre en todo el mundo de aquí a 2030, a combatir las desigualdades dentro de los países y entre ellos, a construir sociedades pacíficas, justas e inclusivas, a proteger los derechos humanos y promover la igualdad entre los géneros y el empoderamiento de las mujeres y las niñas, y a garantizar una protección duradera del planeta y sus recursos naturales (ONU, 2015a: 1).

De acuerdo a la OIT (2016), la Agenda 2030 ubica en el centro a las personas y a los recursos naturales del planeta y hace un llama- 
do a la comunidad internacional a trabajar unida para enfrentar los grandes desafíos de la humanidad, incluyendo los del mundo del trabajo. El Trabajo Decente es el objetivo 8 de esta Agenda.

\section{Método}

Hasta la fecha no existe una metodología única para medir el nivel del Trabajo Decente que sea ampliamente aceptada, tal como sucede con el Índice de Desarrollo Humano (IDH). Lo anterior, debido entre otras cosas a las diferencias en las legislaciones de los países y la cultura laboral. Sin embargo, en los últimos años, en el marco de este programa, se han presentado varias propuestas para medirlo a través de un índice, por ejemplo: Panorama laboral (2001), Ghai (2003), Bescond, Chataignier \& Mehran (2003), Actis (2005), Galhardi (2008), entre otros. Como cualquier trabajo de investigación, todos estos esfuerzos tienen fortalezas y debilidades y a la vez son valiosas aportaciones al estudio del campo laboral. En el presente documento el ejercicio de medición de trabajo decente que se realiza utiliza la metodología propuesta en Gálvez, Gutiérrez \& Picazzo (2011), que representa una aportación más para la medición de la calidad del empleo y cuya construcción se basa en la visión del desarrollo humano y sustentable ya que en la medición se busca reflejar la capacidad de la sociedad de generar empleos que permitan satisfacer al menos las necesidades mínimas necesarias para que las personas puedan vivir dignamente. Esta propuesta puede ser utilizada para hacer análisis a nivel regional o del país, así como en segmentos de población como es este caso donde el análisis se enfoca en las mujeres trabajadoras de Nuevo León. A continuación, se describe la metodología para medir el Índice de Trabajo Decente (ITD).

Para medir la capacidad que tiene una sociedad de generar empleos que permitan a las personas tener al menos un nivel de vida mínimo, cómo señala el desarrollo sustentable, se elaboraron tres indicadores: el empleo durable, el salario suficiente y las prestaciones sociales o equivalente. Sin embargo, antes de definir cada uno de los indicadores es importante señalar que si bien, la OIT señala que el trabajo decente se integra por cuatro dimensiones: el empleo, derechos laborales, prestaciones y diálogo social; en este trabajo se dejará 
para una futura ocasión el último elemento relacionado con el diálogo social, debido a que se busca medir el trabajo decente en las mujeres trabajadoras del estado de Nuevo León en general, y no solo en las asalariadas, que es el único sector en el que pueden organizarse los sindicatos. De acuerdo al artículo 364 de la legislación laboral, "los sindicatos deberán constituirse con veinte trabajadores en servicio activo" (LFT, 2015), es decir, de contemplar este elemento en la medición del índice se estarían excluyendo a las trabajadoras por cuenta propia, a las trabajadoras sin pago y a las asalariadas que laboran en establecimientos con menos de 20 trabajadores, siendo esto de gran importancia pues en el 2013, el 95\% de las unidades económicas del país tenían máximo 10 trabajadores (INEGI, 2016a). Asimismo, es importante señalar que las tres dimensiones que se utilizan están estrechamente interrelacionadas, como lo demuestran los indicadores que se han elaborado y que a continuación se presentan:

\section{Empleo durable}

Este indicador habla de la capacidad de la sociedad de ofrecer empleos para la población en edad de trabajar y que quiere hacerlo, es un indicador sobre la capacidad de generación y fortalecimiento del tejido social de los mercados de trabajo. También se refiere a que los empleos sean una oferta de trabajo en condiciones legalmente codificadas, donde ese sea el tope inferior a partir del cual ningún empleo debe de traspasar. El indicador está compuesto por la conjunción de las siguientes variables:

1) Población ocupada de 18 ańos y más: para este trabajo, la edad de 18 años marca la frontera que separa a la niñez de la juventud, como señala la Convención sobre los Derechos del Niño, auspiciada por la Comisión de Derechos Humanos de la ONU en 1989 y ratificada por México en 1990, que un niño es todo ser humano menor de 18 ańos de edad (Este País, 2009, y ONU, s/f).

2) Población ocupada que percibe al menos un salario mínimo: de acuerdo a la Ley Federal del Trabajo (LFT, 2015), un salario mínimo es la cantidad menor que debe recibir en efectivo el trabajador por los servicios prestados en una jornada de trabajo.

3) Población Económicamente Activa (PEA): es la población ocupada y la población desempleada. Representa la oferta de mano de obra de la sociedad, que demanda o tiene un empleo. 
4) Población No Económicamente Activa (PNEA): que se encuentra disponible para trabajar: Corresponde a los desalentados, es decir, la población que en la semana de referencia no trabajó, ni tenía trabajo, ni buscó uno por considerar que no tenía oportunidad para ello, pero que dijo que aceptaría un empleo si se lo ofrecieran.

Forma de cálculo:

$$
\mathrm{TED}=(\mathrm{PO} / \mathrm{PEAR}) \times 100
$$

Dónde:

TED = Tasa de Empleo Durable

$\mathrm{PO}=$ Población Ocupada de 18 años y más que tiene ingresos mayores de un salario mínimo.

PEAR = Población Económicamente Activa Real, compuesta por la PEA y la PNEA disponible para trabajar.

\section{Salario suficiente}

Este indicador descansa en la idea de que las personas no sólo requieren trabajar sino percibir un ingreso que les permita vivir con libertad y tranquilidad que, como señala Sen (2000), les dé el derecho económico y la libertad fundamental para comprar lo suficiente para vivir. Este indicador toma en cuenta lo siguiente:

a) Población ocupada total.

b) Población ocupada remunerada con ingresos de al menos 3 salarios mínimos, monto que representa el salario suficiente para comprar una Canasta Obrera Indispensable (COI).

Forma de cálculo:

$$
\text { TOSS }=(\mathrm{POI} / \mathrm{POT}) \times 100
$$

Dónde:

TOSS: Tasa de Población Ocupada con Salario Suficiente

PO1 = Población Ocupada con Ingresos Iguales o Superiores al Salario Suficiente

POT = Población Ocupada Total 


\section{Prestaciones sociales o equivalentes}

Toma en consideración que uno de los fundamentos de la idea del trabajo decente es que los empleados cuenten con prestaciones sociales y materiales que les permitan una mayor calidad en el empleo y de vida. En las prestaciones sociales se encuentran los derechos sociales que están ligados a la base trabajadora como el derecho a la salud, a través de los servicios de salud, que aparte de ser un derecho de toda persona que le permite una mayor calidad de vida, fomenta el desarrollo económico. Su forma de cálculo es la siguiente:

$$
\text { TAIS }=(\text { POAIS } / \text { POT }) \times 100
$$

Dónde:

TAIS = Tasa de Acceso a Instituciones de Salud

POAIS = Población Ocupada con Acceso a Instituciones dee Salud Públicas y Privadas

POT = Población Ocupada Total

Para la medición de las prestaciones materiales, se consideran aspectos como vacaciones con goce de sueldo, aguinaldo y demás, que están tipificados en el derecho laboral y permiten a las trabajadoras obtener un ingreso complementario a sus ingresos laborales (se excluye la prestación de acceso a las instituciones de salud) ya sea en dinero o en especie. También se incluye a las mujeres que no tiene prestaciones, pero gozan de ingresos superiores a los 5 salarios mínimos diarios esto, debido a que se considera que su ingreso les permite tener al menos una capacidad mínima de ahorro que de alguna manera compensa la falta de prestaciones. Este sector de empleados puede estar integrado por asalariadas, trabajadoras por cuenta propia y otros. El indicador para su cálculo:

$$
\text { TPOPI }=((\text { POP }+ \text { POSPI }) / \text { POT }) \times 100
$$

Dónde:

TPOPI = Tasa de Población Ocupada con Prestaciones o con Ingresos Altos

POP = Población Ocupada con Prestaciones

POSPI = Población Ocupada Sin Prestaciones y con Ingresos mayores a 5 salarios mínimos. 


\section{POT = Población Ocupada Total}

De esta manera, la medición del indicador de prestaciones sociales o equivalente se hace de la siguiente manera:

$$
\text { POPSE }=3 / 4(\text { TAIS })+1 / 4(\text { TPOPI })
$$

\section{Donde:} valente

POPSE: Población ocupada con prestaciones sociales o equi-

TAIS: Tasa de población ocupada con acceso a instituciones de salud

TPOPI: Tasa de población ocupada con prestaciones o ingresos altos

La ponderación que se asigna a cada componente de este indicador es debido a que se considera que de estos derechos el de mayor importancia es el relacionado al acceso a los servicios de la salud. De esta manera, el indicador construido muestra de forma general qué tanto son respetados los derechos sociales y materiales de las mujeres trabajadoras. Este grupo de indicadores son los que se consideran para medir el ITD de las mujeres trabajadores del estado de Nuevo León. Es claro que no incluyen una visión exhaustiva de todos los aspectos que pueden considerar un trabajo decente. Sin embargo, como señala Sen (2000: 133), muestra algunos de los aspectos más importantes y algunas cuestiones fundamentales desde el punto de vista de la política económica y social que reclaman una atención inmediata.

\section{Aspectos metodológicos para la construcción del ITD y su interpretación}

No obstante, los indicadores descritos y que se van a utilizar para construir el ITD ya se encuentran normalizados, es decir, ya son índices por estar todos expresados en la misma unidad de medida (\%), se estandarizaron a través del método de máximos y mínimos considerando la posibilidad de que en el futuro se agreguen más indicadores los cuales pudieran estar expresados en otras unidades de medición. La propuesta metodológica de máximos y mínimos también es utilizada por el PNUD en el cálculo del Índice 
de Desarrollo Humano (IDH), (PNUD, 2015). Siguiendo esto, el método de conformación del ITD aquí presentado se inscribe en el mismo enfoque de tal suerte que forma parte de la misma frontera de conocimiento. Este método también ha sido utilizado en trabajos como el de Bonnet, Figueiredo \& Standing (2003) y Galhardi (2008). Siguiendo el criterio que emplea el PNUD para la interpretación del IDH, cuando el ITD se ubica en el rango entre 0-0.49 significará un nivel bajo de trabajo decente; 0.50-0.79 indicará un nivel medio de trabajo decente; y de $0.80-1$ un nivel elevado de trabajo decente.

\section{Resultados}

Una vez calculados los índices de cada uno de los indicadores ya descritos, se conformó el ITD quedando como sigue:

Cuadro 1.

Índice de Trabajo Decente y sus componentes para las mujeres trabajadoras del estado de Nuevo León, 2005-2016

Fuente: Elaboración propia con datos de la ENOE/INEGI.

En los datos encontrados para las mujeres trabajadoras del estado de Nuevo León, se puede observar que en el 2005, las condiciones laborales de las mujeres trabajadoras eran mejores incluso que en la actualidad, pues es el único año (de los considerados aquí) en que el ITD se ubicó por encima el 0.5 , es decir, en el nivel medio de trabajo decente. Por componentes se encontró lo siguiente (Cuadro 1):

En el caso del empleo durable, se puede observar que en los años revisados este subíndice se ha mantenido por arriba del 0.6, es decir, en el nivel medio de la escala señalada, además, es el indicador que mejor desempeño ha mostrado de todos los considerados en el ITD. Cabe destacar que el índice de empleo durable cayó en el 2010 a 0.6, con relación al 2005 cuando fue de 0.66 , y desde entonces ha mejorado debido especialmente a que ha disminuido la población femenina que trabaja y no recibe ingresos, así como el número de mujeres menores de edad que trabaja y la cantidad de mujeres desempleadas. Sin embargo, vale la pena señalar que en el periodo de tiempo revisado el año del 2016 destaca por ser el momento en que más mujeres trabajadoras ganan menos de un salario mínimo, condición que se 
encuentra fuera del cumplimiento del código legal del salario mínimo y que además deja a estas mujeres fuera de la posibilidad de contar con un nivel de vida mínimamente aceptable.

La categoría de salario suficiente, es la que peor desempeño tiene de todas las consideradas para el cálculo del ITD. Aunque en el 2005 el indicador registró su nivel más alto, incluso en ese año se ubicó en el nivel bajo de la escala, pero en el periodo estudiado el nivel más bajo fue alcanzado en el 2015 cuando se ubicó en 0.17 , para luego avanzar marginalmente en el 2016. La razón de este pobre desempeño del indicador es que ha habido una disminución constante de la cantidad de mujeres que percibe ingresos mayores a los cinco salarios mínimos, mientras que en el caso de las que ganan de 3 a 5 salarios mínimos se observó un descenso especialmente del 2010 al 2015, cifra que prácticamente se mantuvo en el 2016 (gráfica 1).

\section{Gráfica 1.}

Proporción de mujeres trabajadoras por nivel de ingresos

Nuevo León, 2005-2016

Fuente: Elaboración propia con datos de la ENOE/INEGI.

\begin{tabular}{|l|c|c|c|c|}
\hline \multicolumn{1}{|c|}{ INDICE } & 2005 & 2010 & 2015 & 20.16 \\
\hline Indice de empleo durable & 0.66 & 0.60 & 0.67 & 0.68 \\
\hline Indice población ocupada con salario suficiente & 0.33 & 0.20 & 0.17 & 0.18 \\
\hline Indice población ocupada con prestasiones sociales o equivalent. & 0.55 & 0.53 & 0.57 & 0.59 \\
\hline Indice de Trabajo Decente & 0.513 & 0.475 & 0.470 & 0.428 \\
\hline
\end{tabular}

Además, en el periodo analizado se registró un incremento constante de la proporción de mujeres que gana entre 1 y 2 salarios mínimos, especialmente en el 2015 y 2016), de tal forma que cambió la tendencia de los ingresos percibidos por las mujeres hacia una situación más precaria. Se puede decir, que mientras que en el 2005 y 2010, la mayor parte de las mujeres que trabajaba tenía ingresos de más de 2 hasta 5 salarios mínimos; ahora, entre el 2015 y 2016, la mayor parte de las mujeres percibe ingresos de más de 1 hasta 3 salarios mínimos, situación que va en detrimento de un desarrollo sustentable y del trabajo decente.

Con relación al indicador de prestaciones sociales o equivalente, se encontró que ha ido mejorando marginalmente al paso del tiem- 
po, impulsado especialmente por el aumento de las mujeres trabajadoras con acceso a instituciones de salud y a prestaciones laborales lo que les permite tener una mejor calidad de vida. Sin embargo, el efecto positivo de lo anterior ha sido suavizado por la reducción de la cantidad de mujeres que no tiene prestaciones (ya sea porque no le dan o bien porque trabaja por su cuenta) pero que percibe ingresos mayores de 5 salarios mínimos.

\section{Conclusiones}

Con el análisis realizado se puede decir que en Nuevo León no se está avanzando en el cumplimiento del $8^{\circ}$. Objetivo de la Agenda 2030 del Desarrollo Sustentable en el caso de las mujeres, pues el ITD para este segmento de la población no mejoró en el periodo revisado. Los resultados encontrados mostraron que entre los indicadores considerados, en el índice de los ingresos percibidos, es el que mayormente ha dañado la calidad laboral de las mujeres en el Estado, ya que actualmente el 55\% de ellas gana entre 1 y 3 salarios mínimos; mientras que en el 2005, el $48 \%$ de las mujeres obtenía ingresos de entre 2 y 5 salarios mínimos, además, cada vez son menos las mujeres que ganan ingresos altos, en el 2005, el $15 \%$ de las trabajadoras en Nuevo León tenían ingresos mayores a los 5 salarios mínimos, en el 2016, esa proporción se ha reducido a solamente un $8 \%$. Esto es, en el Estado cada vez hay más mujeres

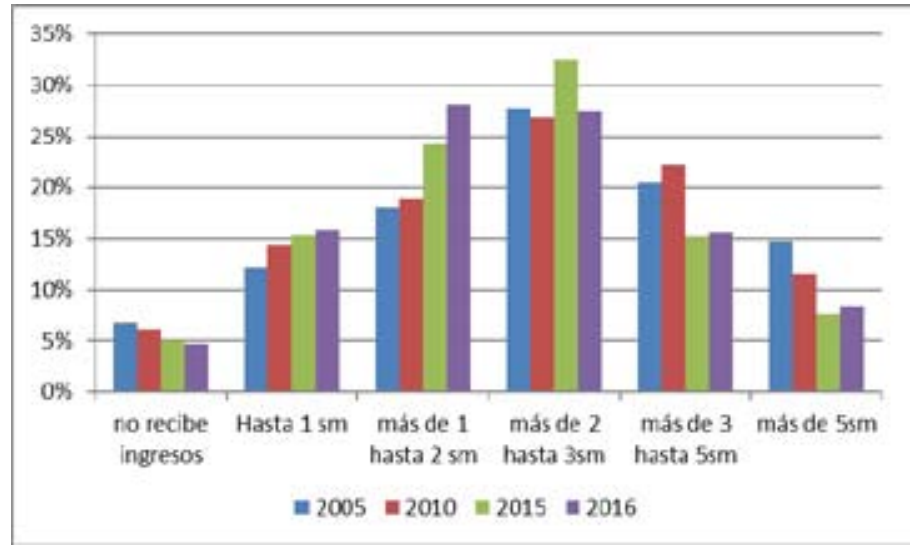

desempeñándose en actividades económicas, el problema es que se 
están incorporando con menores sueldos.

Sin embargo, hay aspectos que muestran que se están haciendo esfuerzos para brindar a las mujeres una mejor calidad laboral y de vida a la vez, tales como un mayor acceso a las instituciones de salud, así como el disfrute de prestaciones materiales; además que cada vez son menos las nińas que se encuentran trabajando. De acuerdo al análisis realizado, desde la visión del trabajo decente y del desarrollo sustentable un aspecto al que se le debe dar más importancia desde las políticas públicas en el Estado es a los ingresos que perciben las mujeres trabajadoras pues son muy bajos, ya que no contar con un ingreso suficiente ocasiona que no se puedan reducir las desigualdades de ingresos afectando no solo lo que las generaciones actuales pueden hacer o ser hoy sino también lo que pueden hacer o ser en el futuro.

Pues, aunque es bueno que nuestro Estado destaque por la importancia de su economía, sólo se hablará de un desarrollo cuando el crecimiento económico sea de calidad, es decir, cuando los beneficios económicos del crecimiento sean disfrutados por las mayorías, en especial por los y las trabajadoras. "El crecimiento económico centrado en el empleo genera un círculo virtuoso que es bueno tanto para la economía como para las personas y, además, impulsa el desarrollo sostenible" (OIT, 2016). Cabe señalar que el estudio realizado no considera situaciones como por ejemplo el trabajo forzoso, la seguridad social, entre otros; sin embargo, permite conocer cómo ha variado la calidad del empleo de las mujeres en el Estado en ciertos aspectos básicos que todo trabajo, formal o informal, debería de cubrir.

\section{Referencias bibliográficas}

Actis, E. (2005). Déficit de trabajo decente en hombres y mujeres. Una aproximación a través de indicadores. Red Académica para el Diálogo Social. Recuperado de http://www.oit.org.ar/documentos/actis_informe_final.pdf

Anand, S.; \& Sen, A. (1994). Desarrollo Humano Sostenible: Conceptos y Prioridades. Documento inédito.

Anker, R., Chernyshev, I., Egger, P., Mehran, F., \& Ritter, J. (2003). La medición del trabajo decente con indicadores estadísticos. Revista 
Internacional del Trabajo, vol 122 num 2, 2003/2. Ginebra, Suiza: OIT.

Bescond, D., Chataignier, A. \& Mehran, F. (2003). Siete indicadores para medir el trabajo decente. Comparación Internacional. Revista Internacional del Trabajo, vol. 122, no. 2: 197-231. Ginebra, Suiza: OIT.

Bonnet, F., Figueiredo, J. \& Standing, G. (2003). Una familia de índices de trabajo decente. Revista Internacional del Trabajo, vol. 122, no. 2: 233-261. Ginebra, Suiza: OIT.

Comisión Mundial sobre el Ambiente y el Desarrollo (CMMAD), (1987). Nuestro Futuro Común. Recuperado de http://worldinbalance.net/intagreements/1987-brundtland.php

Este País (2009). Niñez Trabajadora. Magnitud y características del trabajo infantil en México. Revista Este País, num. 219. junio.

Galhardi, R. (2008). Indicadores de trabajo decente: Un índice para México. Oficina para Cuba y México: Organización Internacional del Trabajo.

Gálvez E., Gutiérrez, E., \& Picazzo, E. (2011). El trabajo decente: nuevo paradigma para el fortalecimiento de los derechos sociales. Revista Mexicana de Sociología, vol. 2011, no. 1, México. UNAM.

Ghai, D. (2003). Trabajo decente. Concepto e indicadores. Revista Internacional del trabajo, vol. 122, num. 2: 125-160, OIT, Ginebra, 2003.

Gutiérrez \& González, E. (2010). De las teorías del desarrollo al desarrollo sustentable. México, DF.: Siglo XXI-UANL.

INEGI (2016). Encuesta Nacional de Ocupación y Empleo. Recuperado de: http://www.inegi.org.mx/est/lista_cubos/consulta. aspx?p=encue $\& \mathrm{c}=4$

INEGI (2016a). Estructura del sector privado y paraestatal por empresas que realizaron actividades en 2013, según actividad, tipo y tamaño de la empresa. Recuperado de: http://www.beta.inegi.org.mx/proyectos/ce/2014/

LFT (2015). Ley Federal del Trabajo. Recuperado de: http://www.diputados.gob.mx/LeyesBiblio/pdf/125_120615.pdf

Lozano, D., et. al. (2006). Necesidad de un programa de recuperación del poder adquisitivo del salario en México. Reporte de Investigación núm. 70, Centro de Análisis Multidisciplinario (CAM) de la Faculta de Economía de la UNAM. Recuperado de: http://www.economia. 
unam.mx/cam/index.html

OIT (1999). Memoria del director general: Trabajo Decente. 87a. Reunión de la Conferencia Internacional del Trabajo, Ginebra, junio 1999. Recuperado de: http://www.ilo.org/public/spanish/standards/ relm/ilc/ilc87/rep-i.htm

OIT (2016). Trabajo decente y la agenda 2030 de desarrollo sostenible. Recuperado de: http://www.ilo.org/global/topics/sdg-2030/lang-es/index.htm

ONU (s/f). Convención sobre los derechos de los niños. Recuperado de: http://www.unhchr.ch/spanish/html/menu3/b/k2crc_sp.htm

ONU (2015). Objetivos de Desarrollo Sostenible. Recuperado de: http:// www.un.org/sustainabledevelopment/es/economic-growth/

ONU (2015a). La Asamblea General adopta la Agenda 2030 para el Desarrollo Sostenible. Recuperado de: http://www.un.org/sustainabledevelopment/es/2015/09/la-asamblea-general-adopta-la-agenda2030-para-el-desarrollo-sostenible/

ONU (2016). Desarrollo Sostenible. Recuperado de: http://www.un.org/ es/ga/president/65/issues/sustdev.shtml

Panorama Laboral (2001). Índice de desarrollo de trabajo decente 19902000. Panorama Laboral 2001, Regional para América, Lima, Perú, diciembre.

PNUD, 2000. Informe sobre desarrollo humano 2000. Consultado en: http://hdr.undp.org

PNUD, 2015. Reporte del Desarrollo Humano 2015. Recuperado de: http://hdr.undp.org/sites/default/files/hdr_2015_report_sp.pdf

Sen, A. (2000). Desarrollo y Libertad, Barcelona, Editorial Planeta. (Traducción del Development as Freedom (1999), London, Oxford University Press). 